\title{
Concordance for Amyotrophic Lateral Sclerosis in a Pair of Dizygous Twins of Consanguineous Parents*
}

\author{
J. DUMON, J. MACKEN, and TH. de BARSY $†$
}

From the Department of Neurogenetics of the Born-Bunge Foundation, Berchem-Antwerp, Belgium

The classical form of amyotrophic lateral sclerosis (ALS) or Charcot's disease contains both familial and sporadic cases. The familial form has been extensively reviewed by Faveret (1959), Haberlandt (1964), and Becker (1966). Sporadic cases present the same clinical features. In addition, there is a form of the disease encountered in the native inhabitants of Guam and Japan. It displays several clinical signs and pathological lesions identical to those of the classical form, and yet it shows differences concerning epidemiology, age of onset, and sex ratio (Hirano et al., 1967; Yase, 1966; Kurland, 1965). The two patients to be presented show the clinical features of the classical type of ALS. Though nosologically unremarkable, they are of interest because they are dizygotic twins and the products of a consanguineous marriage.

\section{Case Histories}

Case 1. R.V. 46 years of age, father of four healthy children, is the son of a first cousin marriage. The family history (Fig.) is otherwise unremarkable. He has enjoyed excellent health until the age of 44 when he first noted difficulties in mounting stairs. His gait became laborious due to progressive weakness first of the right leg and subsequently of the left one. In March 1965 he was seen as an out-patient in the Department of Neurology of the Bunge Institute, Antwerp, Belgium. At that time he complained about severe pain and weakness in the right leg. An electromyographic examination (EMG) was performed by Dr. C. Claes. The record at rest showed signs of denervation in the right quadriceps and tibialis anterior muscles. Moreover at maximal contraction, a loss of motor units could be demonstrated. The mean duration of action potential was raised by 20 to $35 \%$. The findings were interpreted as consistent with a pluriradicular lesion, though the presence of anterior horn pathology could not be ruled out. During the subsequent months the condition progressed to the point that patient could no longer walk without the use of

\footnotetext{
Received 24 April 1970.

* This work was supported by the Belgian Medical Scientific Research Foundation (Genetic Program).

t Aspirant of N.F.W.O. of Belgium.

8-J.M.G.
}

a stick. The patient himself had also noticed wasting of the lower limbs particularly on the left side, diminution of strength in the upper limbs, and discrete dysphagia as well as an alteration of the voice.

In April 1966 he was admitted for re-evaluation at the Department of Neurology of the Bunge Institute. Clinical examination revealed a waddling and paretic gait with genu recurvatum, an inferior facial paralysis on the right side, and a homolateral paresis of the palate. There were bilateral lingual fasciculations. Weakness of the hands and proximal weakness of the lower extremities extending to the abdominal musculature were readily apparent. Fasciculations were mainly proximal in the upper limbs and more diffusely spread in the lower ones. The deep tendon reflexes of the upper extremities were brisk with a paradoxical triceps reflex on the right. On the contrary, the patellar reflexes were weak and the triceps surae reflexes unobtainable. Babinski's sign was present bilaterally, the Hoffman reflex only on the left side. Abdominal and cremaster reflexes were present. Touch, pain, temperature, and proprioceptive sensitivity were within normal limits, as were all cerebellar functions.

Routine studies of urine and blood, including serum enzymes, yielded normal results except for raised $\gamma$-globulin concentration revealed by agar electrophoresis (22\% compared to the normal range from 14 to $18 \%$ ). Cell and total protein content of the cerebrospinal fluid were normal as was protein electrophoretic pattern on agar.

A second EMG examination during this admission showed a severe degree of denervation with mean potential duration increased by as much as $25 \%$ to $40 \%$. This time a very progressive lesion of the anterior horn cells was thought to be the reason. Because of the history, the clinical signs, and the EMG findings, a diagnosis of ALS was made at the time of discharge.

Case 2. P.V. is the twin brother of R.V. and has an unremarkable past history. The onset of his disease occurred soon after that in his brother. In August 1965 he started complaining about low back pain. This was soon followed by dragging of the right leg and motor impairment of the left arm. By January 1966, similar disabilities had become apparent in the left inferior and right superior limbs. In addition he suffered from 


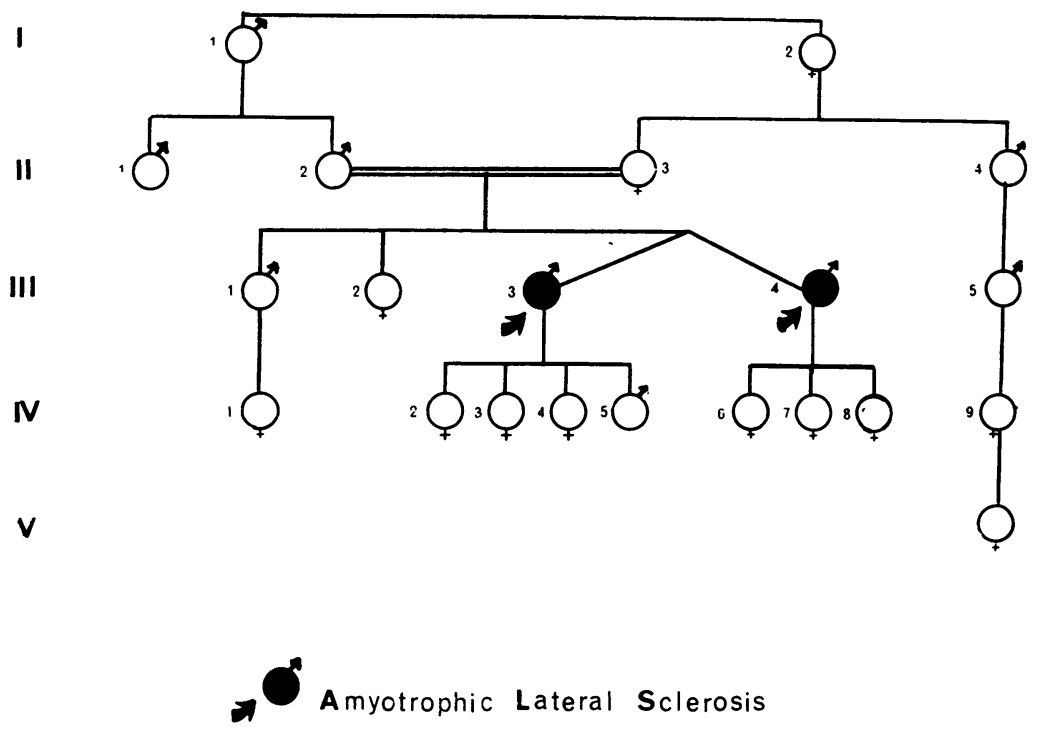

FIG.

muscular pains of variable severity and he noted constant fasciculations. Following the diagnosis made in his brother, P.V. was seen in the neurological outpatient Department of the Bunge Institute. He presented with a bilaterally spastic and wadding gait. Important muscle wasting was obvious in all four extremities, predominating however in the left arm and in the right leg. Muscle weakness was mainly distal. Numerous fasciculations were present over the entire body, including twitching of the lingual musculature. There was also a paresis of the soft palate on the right side. The deep tendon reflexes were brisk everywhere, and Babinski's sign was present bilaterally. The abdominal reflexes were normal and no impairment of sensitivity or cerebellar functions could be demonstrated. Though this patient refused all proposed complementary investigations, the diagnosis of ALS was obvious because of the brother relationship and the consistent clinical picture.

Studies on the zygosis of this pair of twins were guided in the Department of Neurogenetics of the Bunge Institute. From the blood group studies, performed in two independent laboratories, it was concluded that the twin pair under study was dizygous. The results are summarized in the Table. Physically the twins are dissimilar. Also psychologically they

\section{TABLE}

BLOOD GROUPS IN PRESENT TWINS*

\begin{tabular}{|c|c|c|c|c|c|}
\hline $\begin{array}{l}\text { Case } 2 \\
\text { Case } 1\end{array}$ & $\begin{array}{l}\mathrm{O} \\
\mathrm{O}\end{array}$ & $\begin{array}{l}\text { CcDee } \\
\text { CcDee }\end{array}$ & $\begin{array}{l}\text { MNSs } P(1-) \\
\text { NNss } P(1-)\end{array}$ & $\begin{array}{l}\text { kk Fy(a-) } \\
\text { kk Fy(a-) }\end{array}$ & $\begin{array}{l}\mathbf{J k}(\mathbf{a}+) \\
\mathbf{J k}(\mathbf{a}+)\end{array}$ \\
\hline
\end{tabular}

differ considerably. The results of the dermatoglyphic studies would have been equivocal, if interpreted without the data on blood groups. The total fingerprint ridge count, usually a reliable dermatoglyphic trait in twin diagnosis, yielded an intrapair difference of only 6 ridges. According to Smith and Penrose (1955), this small difference would be more indicative of monozygosity. Secondly, the many differences observed in digital ridge patterns, in morphology of palmar areas, and in main line endings, could be ascribed equally well to a pair of a monozygous as to a pair of dizygous twins. Thirdly, there was an absence of both $\mathrm{D}$-line and triradius $d$ in the left hand of R.V. This peculiarity is extremely rare in the general population (Cummins and Midlo, 1943), and since it is found in one twin only, it argues in favour of dizygosity.

Unfortunately it was not possible to examine other members of the family.

\section{Discussion}

Because the two patients presented, both having the symptoms and signs of the classical form of amyotrophic lateral sclerosis, the current controversy on whether or not all clinical forms of ALS represent a single condition is irrelevant. There is also a difference of opinion on the aetiology of ALS. Environmental factors, such as nutritional deficiencies, toxic substances, and various types of viral infections, have been proposed as possible causes (Kurland, 1965). On the other hand, heredity has always been considered important in this respect. Two facts should be noted in our patients for separate discussion: first, the two patients are twins; 
next, they are the sons of a consanguineous marriage.

Since the patients are dizygous twins, the observed concordance for ALS can equally point to environmental as to hereditary causal factors. Only a large series of twins with the same clinical form of ALS can contribute to the solution of this dilemma. Significantly more concordance in monozygotic twins than in dizygotic ones would favour an hereditary basis of the condition, whereas equal concordance in both kinds of twins indicates an environmental cause. The literature on ALS in twins is obviously scarce. Unfortunately, it is also unreliable. A thorough search has found the description of only five pairs of twins with a tentative diagnosis of ALS in one or both of them. Twin sisters affected from age 33 with a slowly evolving neurological disease were described by Salus (1935). Both showed progressive motor impairment with all clinical features of ALS. No information is available on the zygosity of those twins. Haberlandt (1964) reports on four pairs of twins in relation to ALS, but he concedes that no useful information can be drawn from those observations. In two of the four pairs one twin died in infancy. One member of the third pair could not be examined. The fourth pair is said to be monozygous. In this set of twins, the diagnosis of ALS can be doubted because they were concordant for a progressive bulbar paralysis associated with palpebral ptosis and opthalmoplegia. An ocular myopathy cannot be ruled out in this case (Becker, 1966). On an a priori basis, ALS in one or both members of a set of twins is a rare event. We therefore thought it of interest to communicate our observation. Similar reports by others might provide in the future a series of twins sufficiently large to assign proper weights to hereditary and environmental factors in the aetiology of this condition. The twins presented are the sons of a first cousin marriage. No other members of the family are affected or are said to have any neurological disease.

In particular, no evidence was obtained of Parkinsonism and dementia, conditions known to be sometimes associated with ALS. In the literature a few instances are known of ALS patients with a coefficient of inbreeding exceeding that of the general population. Haberlandt (1964) cites two pedigrees. In one the parents were first cousins; in the other one the degree of parental consanguinity could not be determined accurately. Becker (1966) reports on two sisters with ALS whose parents were first cousins once removed.

Our observation and the above facts from the literature could be consistent with an autosomal recessive mode of inheritance of the condition, provided a definite hereditary basis can be established.

On the other hand autosomal dominant transmission with incomplete penetrance is the impression derived from the familial cases in general. If the 'formes frustes' can be accepted as really representing ALS, the responsible mutant gene would show a variable phenotypic expression.

It is possible that the form of ALS described (Kurland, 1965; Plato et al., 1967; Hirano et al., 1967) in many inhabitants of Guam represents a different clinical entity. It is as yet impossible to settle the question on its environmental or hereditary aetiology. Assuming the latter to be true an autosomal dominant transmission of the trait would be the more likely mode of inheritance in the highly inbred population of the village of Utamac, Guam (Plato et al., 1967), and in the patients of the Kii Peninsula, Japan, where parental consanguinity in the cases could be detected (Yase, 1966). From the analysis of the pedigrees of ALS patients, the possible mode of inheritance of the syndrome is at least equivocal. This can be due to the fact that cause is purely environmental. It could also be consistent with the contention that there are two or more distinct clinical forms with a different mode of inheritance. An interaction of an environmental factor such as a viral infection with chronic implication and an especially susceptible genetic make-up is an attractive hypothesis but is hard to put to a rigorous test. Twin data could become very useful here, and this consideration was the stimulus to us for reporting the ALS in this pair of dizygotic twins.

\section{Summary}

Well-documented amyotrophic lateral sclerosis (ALS) is reported in twin brothers. The pair of twins is dizygotic. They are the products of a first cousin marriage. The relevance of this observation to the controversy on the aetiology and mode or inheritance of ALS is briefly discussed.

\section{REFERENCES}

Becker, P. E. (1966). General editor. Krankheiten des Nervensystems. By P. E. Becker and G. Koch. Humangenetik, Vol. 5, Part 1. Georg Thieme, Stuttgart.

Cummins, H. and Midlo, C. (1943). Finger-prints, Palms, and Soles. An Introduction to Dermatoglyphics. Blakinston, Philadelphia.

Faveret, C. (1959). La Sclérose Latèrale Amyotrophique Familiale. Thesis, de Paris. Vézin, Paris.

Haberlandt, W. F. (1964). Amyotrophische Lateralsklerose, 185 pages. Gustav Fischer, Stuttgart.

Hirano, A., Arumugasamy, N., and Zimmermann, H. M. (1967a). Amyotrophic lateral sclerosis. Archives of Neurology, 16, 357363.

- Kurland, L. T., and Sayre, G. P. (1967b). Familial amyotrophic lateral sclerosis. Archives of Neurology, 16, 232-243. 
Kurland, L. T. (1965). Amyotrophic lateral sclerosis: a reappraisal. In Slow, Latent and Temperature Virus Infections, pp. 13-22. Ed. by D. C. Gajdusek, National Institute of Neurological Diseases and Blindness, Washington.

Plato, Ch. C., Reed, D. M., Elizan, T. S., and Kurland, L. T. (1967). Amyotrophic Lateral Sclerosis/Parkinsonism-Dementia Complex of Guam. IV. Familial and Genetic Investigations. American fournal of Human Genetics, 19, 617-632.
Salus, F. (1935). Beiträge zur Lehre von der amyotrophischen Lateral-sklerose. Ein Erklärungsversuch über die endogene Entstehungsweise. Archiv für Psychiatrie und Nervenkrankheiten, 104, 66-88.

Smith, S. M. and Penrose, L. S. (1955). Monozygotic and dizygotic twin diagnosis. Annals of Human Genetics, 19, 273-289.

Yase, Y. (1966). A study of amyotrophic lateral sclerosis. Wakayama Medical Reports, 11, 125-128. 